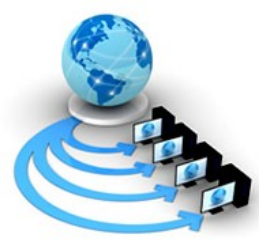

Volume 10, No. 1, January-February 2019

ISSN No. 0976-5697

International Journal of Advanced Research in Computer Science

RESEARCH PAPER

Available Online at www.ijarcs.info

\title{
INTRANET BASED WORKFORCE MANAGEMENT SYSTEM FOR SPI CUSTOMER RELATIONSHIP MANAGEMENT, INCORPORATED
}

\author{
Carlo A. Batitis \\ College of Computer Studies \\ Trimex Colleges, Biñan, Laguna, Philippines \\ Joseph I. Pilar \\ College of Computer Studies \\ Trimex Colleges, Biñan, Laguna, Philippines
}

\author{
Ermilo S. Poso \\ College of Computer Studies \\ Trimex Colleges, Biñan, Laguna, Philippines \\ Ma. Cecilia M. Balboa \\ College of Computer Studies \\ Trimex Colleges, Biñan, Laguna, Philippines
}

\author{
Louie F. Agustin \\ College of Computer Studies \\ Trimex Colleges, Biñan, Laguna, Philippines
}

\begin{abstract}
Intranet-Based Workforce Management System for SPi Customer Relationship Management Inc., Makati City is the proposed system that provides employee information, agent schedules, updated team roster and reports to users which could be a process improvement for workforce management team and human resources department when it comes to validation of requests from operations. As a whole system, it provides information for the agent and users in-terms of schedules and reports that can be useful to assess performance. It also has useful functionalities that help to improve the current process of workforce management when it comes to record-keeping and data processing.
\end{abstract}

Keywords: Process management; Workforce Management; Information systems applications; Time Management

\section{INTRODUCTION}

Changes in innovation bring significance and headway in our lives. From a modern culture, the researchers are experiencing a significant change to a data society. Innovation has changed the manner in which how individuals function, work together, tackling issues, do look into, making the method for correspondence less demanding and the assortment of decision that the general population needs to discover in a diversion. The development of innovation truly affects how business run and functions today, particularly in the business process industry and in workforce management. Business Process Outsourcing consequently or generally alludes to the rearrangement of full business capacities to some other service providers, fundamentally in low-cost locations. The service provider might be either self-claimed or a third party. For a BPO company like SPi Customer Relationship Management Inc., Workforce management is essential since it helps the organization to maximize employee productivity level, improve time management, monitors resources, and the entire process.

The primary job role of a workforce specialist is scheduling, planning, forecasting of workload, adherence monitoring, process improvement, optimization of staffing level, record keeping and data processing. With this duties and responsibilities, workforce management and HR department uses different kinds of tools such as MS Excel and MS Outlook for reporting, Blue Pumpkin for scheduling and forecasting, WISE for employee information and leave tracker, HRPRO for payroll processing and reports, MS Sharepoint for schedule change requests and CosmoCall Universe for agents time-in and time-out. Both departments have difficulties on updating, keeping and processing information from this system such as team roster, attendance, and attrition report, schedule change requests, agents time-in, and time-out and employee headcount due to its traditional way of putting records of lists. Through these challenges, the researchers will develop and design a proposed system that will help to incorporate common information and process different kinds of operational requests. SPi Customer Relationship Management Inc. Citimotors located at $6^{\text {th }}$ floor Citimotors Bldg., Chino Roces Avenue, Makati City. 


\section{DESIGN OF THE STUDY}

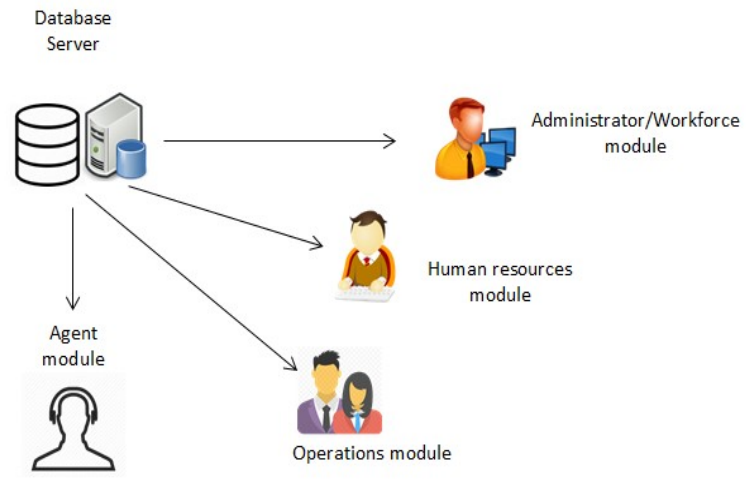

Figure1: System Architecture

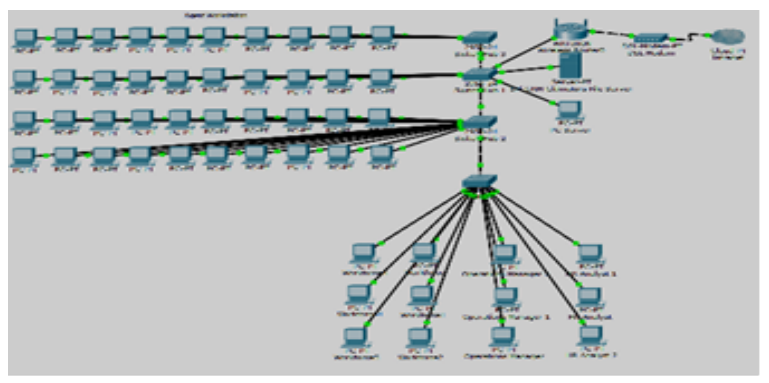

Figure 2: Network Design Infrastructure

The figure demonstrates the sample network connectivity of the workforce department, HR department, and operations. This serves as the file server for operations that will be used to cater the workforce management support gateway system. Most of the hardware components used are core i5 models with Windows 7 as the main operating system for each unit.

The figure demonstrates the sample network connectivity of the workforce department, HR department, and operations. This serves as the file server for operations that will be used to cater the workforce management support gateway system. Most of the hardware components used are core i5 models with Windows 7 as the main operating system for each unit.

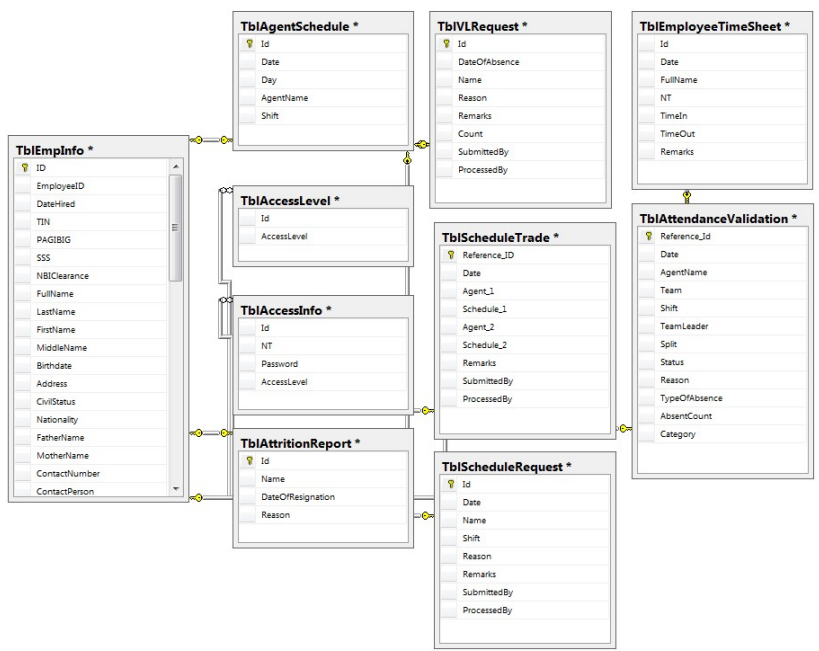

Figure 3. Database Schema

This figure demonstrates the database design of the proposed system. The database name is SPICRM_WFM and consists of 10 tables.

\section{LITERATURE SURVEY}

\section{Indian Institute of Management Indore}

An administrations association's gainful development is reliant on its capacity to have the correct number of individuals with right capabilities in the correct area at the perfect time at the ideal expense. Powerful workforce administration (WFM) choices at key, strategic and operational level are necessary to accomplish this. Operational decisions incorporate the coordinating and task of the workforce to the undertaking positions. Strategic arranging alludes to medium term basic leadership to decide the number, ability blend, area and experience groups of the workforce to be kept up with the end goal to take into account the interest (venture positions) in an arranging skyline of multi-month, a quarter or a year. This incorporates choices identified with enlisting, preparing and terminating of workforce amid an arrangement period. At the vital level, human asset and business arrangements which control operational and strategic arranging choices are produced, assessed and chose. Indian Information Technology (IT) administrations industry income was 146 billion US Dollars in the fiscal year 2015 with a development of $13 \%$ year on year. With the automation difficulties and protectionism in the significant markets, the Indian IT administrations industry needs creative WFM ways to deal with proceeding with its development story. Selvaraj Vadivelu(October 2017) Workforce management decisions in IT services organizations.

\section{Managing a Global Workforce: Challenges and Opportunities in International Human Resource Management}

Organization pioneers attempt to deal with their associations inside a developing worldwide market setting, with quickly evolving social, political, monetary, and innovative powers. Inside this global setting, pioneers confront 
the need to help out their work exercises through the endeavors of their nation of origin representatives and also their outside workers, specialists, association accomplices, and providers as hierarchical limits turn out to be more penetrable and less unmistakable with new work connections and community-oriented understandings. For example, key unions, global joint endeavors, and redistributed administrations, and with relating new, more adaptable workforce game plans. For instance, low maintenance and impermanent representatives and contracted work benefits, the view of what establishes an association's workforce should be likewise balanced.

Consequently, this evolving workforce, which is necessary for accomplishing authoritative objectives and destinations, is ending up more worldwide, different, adaptable, multi-sourced, and involved in nature. This world situation shows endlessly excellent chances and requests and progressively troublesome difficulties than were even looked toward the finish of the twentieth century. Charles M. Vance, Yongsun Paik(2015).

Employee Motivation and Productivity: A review literature and implications for management practice

This study is likewise convenient in the Nigerian economy with the present retreat portrayed by low representative spirit, low deals volumes, difficulties to mechanical amicability, and so on. It was contended that inspiration is the way to monetary recuperation in the nation. A survey of hypotheses and experimental proof on the focal issues in question on the most proficient method to help inspire in this manner turns out to be convenient. Whatever was left of the paper is partitioned into four segments. The prompt section after this presentation quickly surveys a few speculations and observational examinations on inspiration. The third area shows our perspectives on the motivation of laborers for upgrading their profitability depends on the writing examination. Area four finishes up the paper. $\operatorname{Aremu(2017).~}$

\section{Workforce Management}

Workforce management doubtless alters the optimization of the control of the force of a service supplier to maximize each service quality and potency. Though the extent analysis literature provides characterizations of the varied typologies of WFM systems, the results of those systems on productivity and condition wasn't investigated extensively. The Impact of Workforce Management Systems on Productivity and Quality: A Case Study on the Information and Communication Technology Service Industry. Armando Calabrese, Guendalina Capece, Roberta Costa Francesca Di Pillo, Daniela Paglia(August 19, 2013).

Our workforce and our whole economy are most grounded after we grasp decent variety to its fullest, which implies that hole entryways of opportunity to everyone and perceiving that the American Dream bars no one. Thomas Perez(May 21, 2017).

A standout amongst the best courses for pioneers to expand representative commitment is to guarantee that the workplaces and societies that they manufacture empower their kin to fulfill their PEMS needs. The arrangements are regularly misleadingly necessary. For instance, one supervisor who participated in Ruben's instructing program was in charge of a profoundly withdrew worker who might not attempt his best to build up the PC aptitudes that he expected to play out his duties and would reliably submit expectations of low quality. Benito L. Teehankee(October 4, 2016).

\section{RESULT AND DISCUSSION}

\section{Employee Information}

The figures below show that the system has employee information feature and is authorized to use by workforce management and HR department to add, update and generate records.

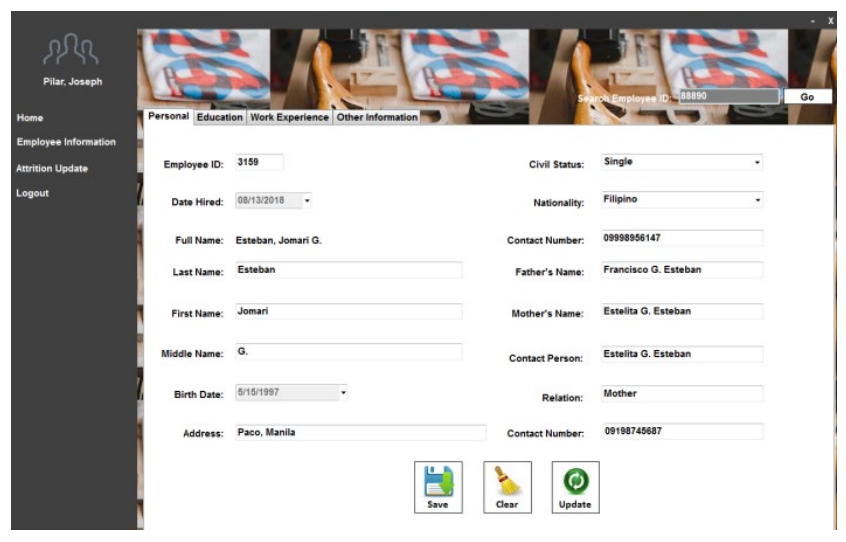

Figure 4: Employee Information Attrition Update

Below figure shows the attrition menu and the report for attrition count. It is being reported by operations and processed by HR Department thru attrition menu to have the accurate information and can also be used by workforce management on its hiring plan.

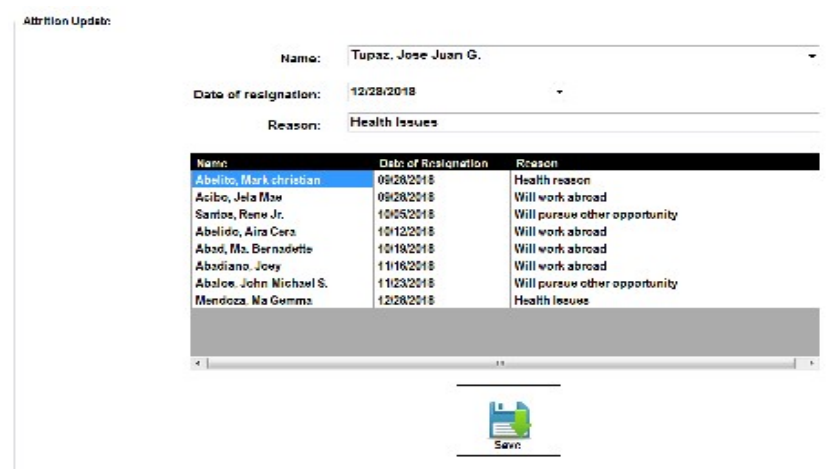

Figure 5: Attrition Update Time-in and Time-out

The figure below shows the time-in and time-out. Agents are required to time in and time out during the start and end of their shift. All records are stored in the database. 


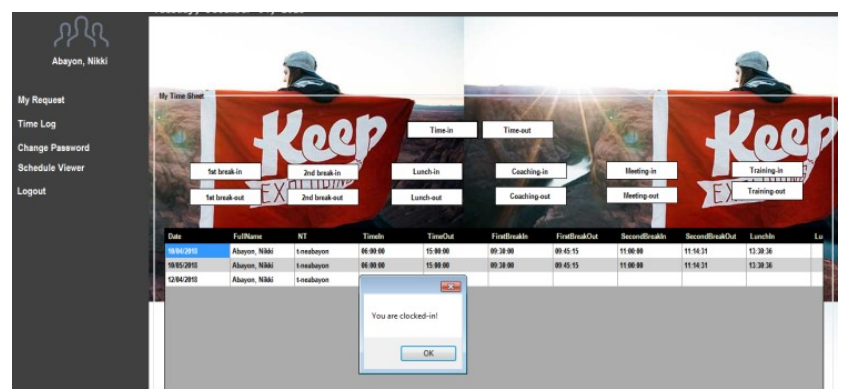

Figure 6: Time-in and Time-out Agent Schedules

The figure below shows the generated schedule of the agents. The WFM team can upload it from the external tool, and it can be accessed and viewed by users.

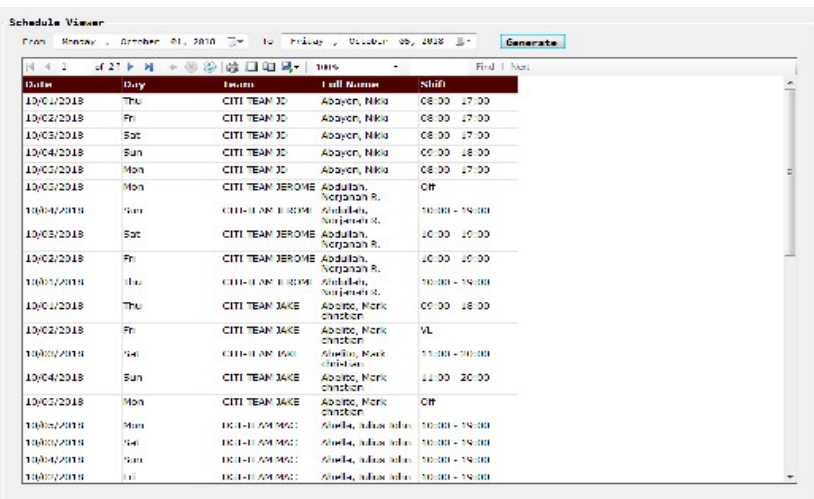

Figure 7: Agent Schedule Time-in and Time-out Report

Below report shows the timesheet. Once an agent timein or time-out, it will be recorded in the database and reflected in the report.

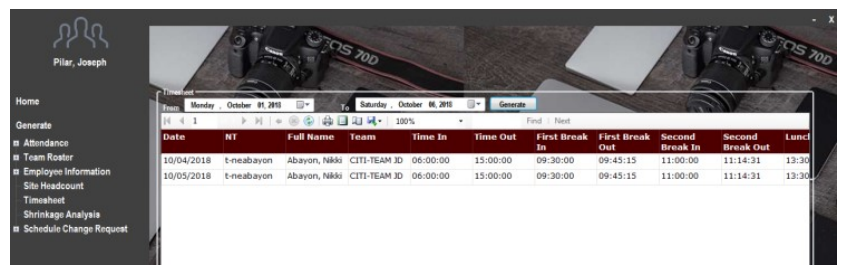

Figure 8: Time-in and Time-out Report Attendance Report

The figure below shows the report for attendance. Supervisors have access to update the status of their agents, and will reflect in the report at the same time.

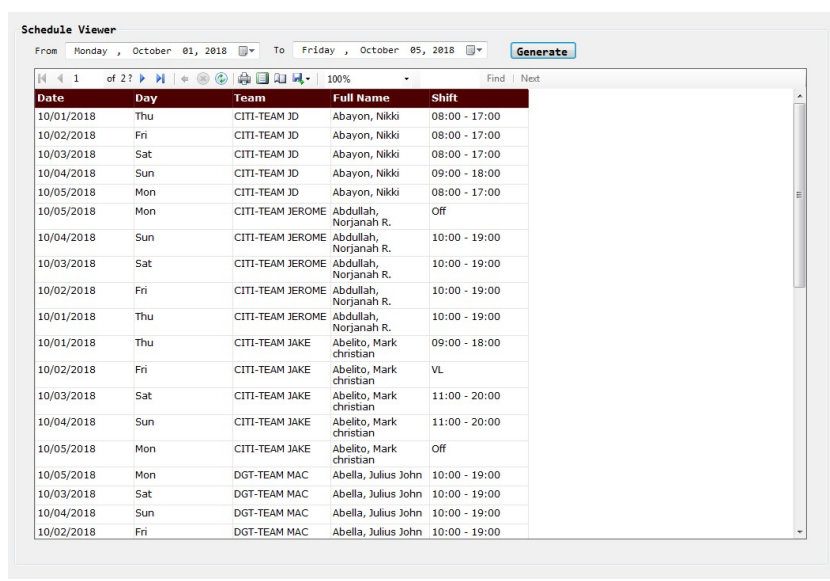

Figure 9: Attendance Report Shrinkage Analysis

Below figure shows the Shrinkage Analysis Report. It is automatically processed by the system by counting off the total agents on-leave divided by the total scheduled FTE for each day.

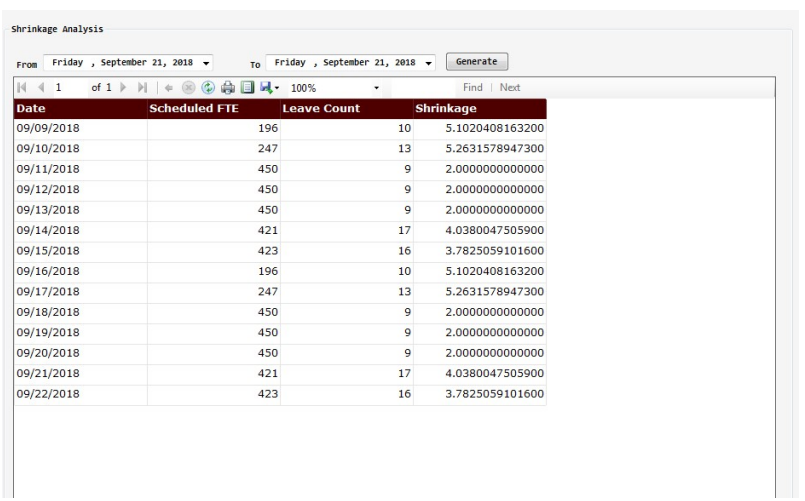

Figure 10: Shrinkage Analysis Headcount

Below figure shows the generated report for site headcount. All employees that are tagged as resigned by HR department are excluded in this report. The system is automatically counting off the active employees only.

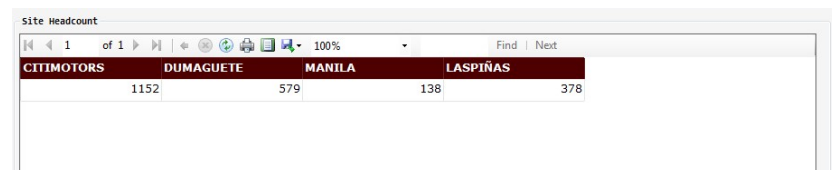

Figure 11: Headcount Schedule Change Requests

Based on the current process, all schedule change requests are forwarded by supervisors thru e-mail but using the proposed system, now it has a tool that can process the request easily. Below figure shows the process for different schedule change requests. The supervisors are authorized to submit the request of their agent and will automatically move to approval stage care of workforce department. 


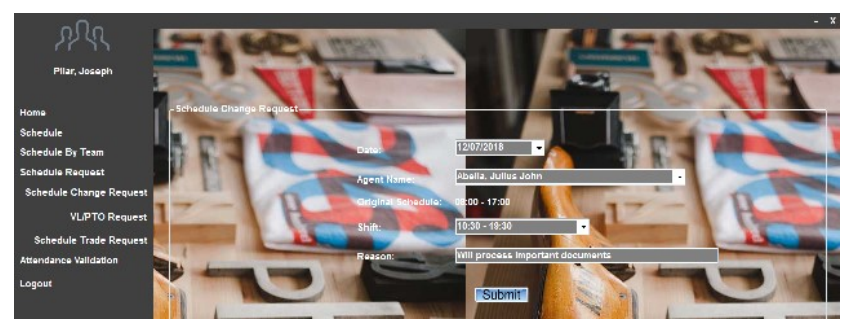

Figure 12: Schedule Change Request

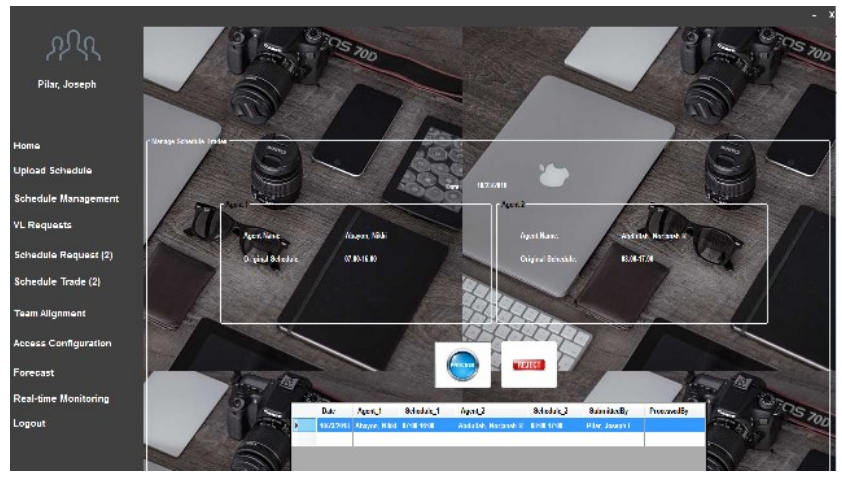

Figure 13: Schedule Trade

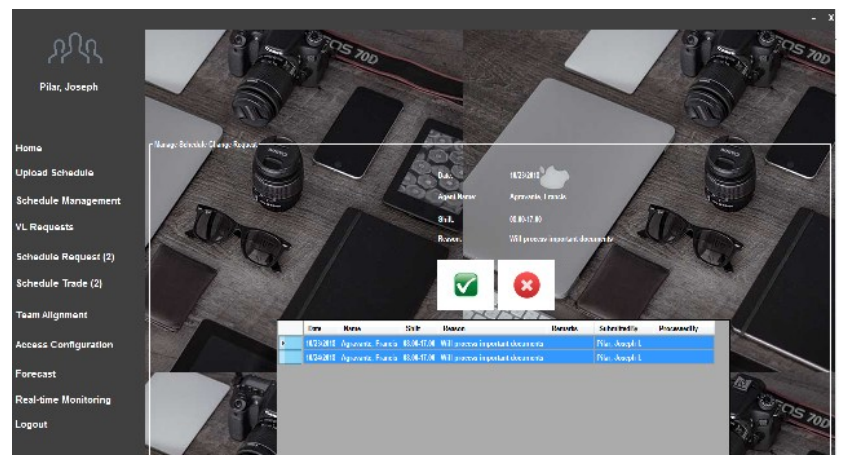

Figure 14: Schedule Change Attendance Validation

The figure below shows the attendance validation feature of the system. Team leaders or even operations manager is responsible for updating the attendance status of their team members

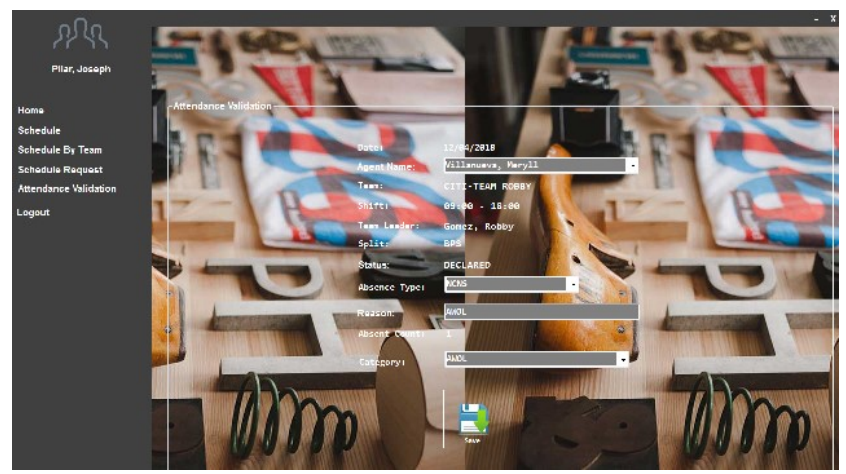

Figure 15: Attendance Validation Active Team list
Below figure shows the process of updating team roster. Operations are having weekly or monthly team alignment and they are sending team alignment request. All updates are being processed by Workforce Management. All employees that are tagged resigned by HR Department are no longer available to update in this feature.

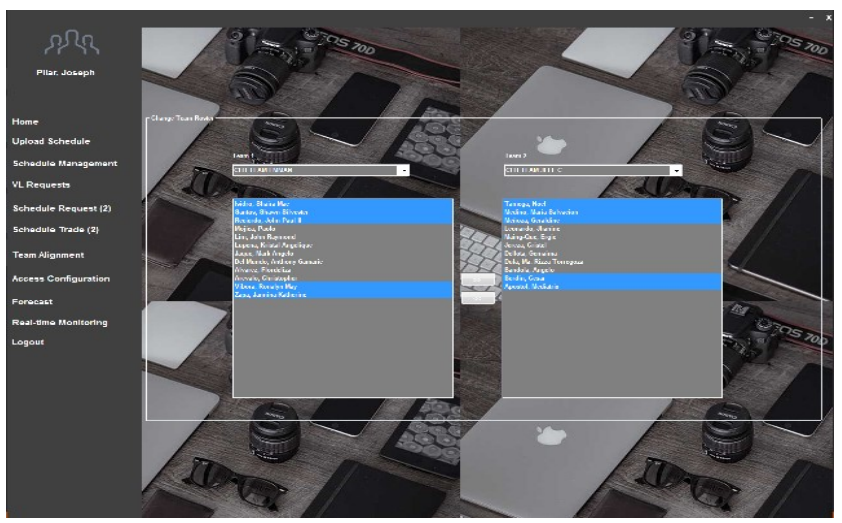

Figure 16: Active Team list Create schedule

Below figure shows the process of creating a schedule. Workforce management scheduler will select a date, agent name, campaign, task, start and end time then the system will record the schedules in the database

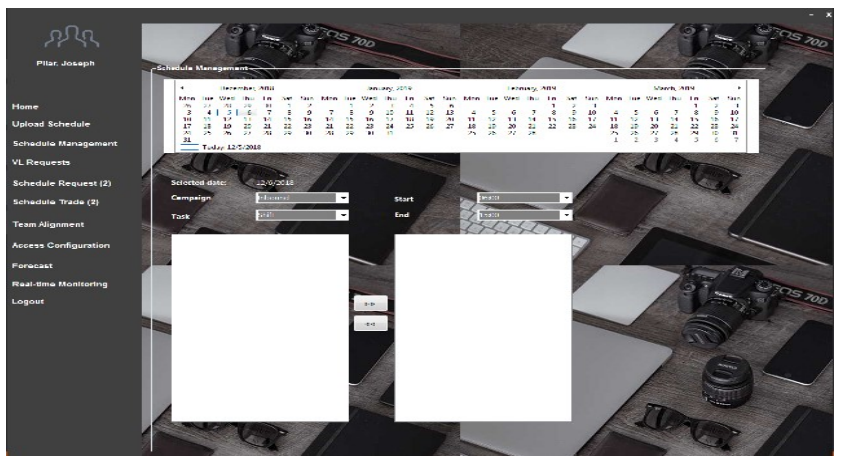

Figure 17: Create Schedule Forecast volume

Below figure shows the process of generating forecasted volume. Workforce management specialist will input the volume for the last two weeks then the system will average the two volumes and it will generate the required fulltime equivalent.

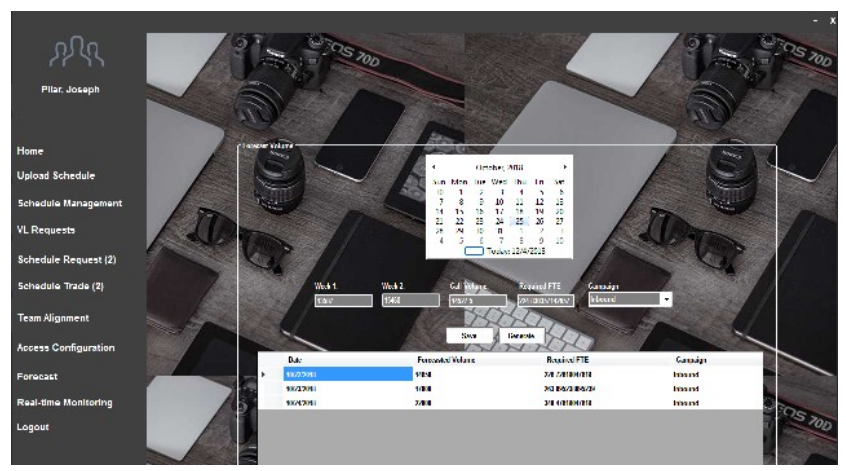

Figure 18: Forecast volume 


\section{CONCLUSIONS}

Workforce Management Support Gateway gives a modern bundle of contact-center administration features, empowering contact focus directors and administrators to all the more likely deal with their workforce. It is intended to fuse and process constant or recorded data that has a contact with business activities.

\section{REFERENCES:}

[1] Selvaraj Vadivelu(October 2017) Workforce management decisions in IT services organizations.

[2] Charles M. Vance, Yongsun Paik(2015), Aremu(2017), Thomas Perez(May 21, 2017).

[3] The Impact of Workforce Management Systems on Productivity and Quality: A Case Study on the Information and Communication Technology Service Industry. Armando Calabrese, Guendalina Capece, Roberta Costa Francesca Di Pillo, Daniela Paglia(August 19, 2013).

[4] Benito L. Teehankee(October 4, 2016). 